\title{
Dementia and Firearms: an Exploratory Survey of Caregiver Needs
}

\author{
Marian E. Betz, MD, MPH ${ }^{7}$, Megan L. Ranney, MD, MPH', \\ Christopher E. Knoepke, PhD, MSW ${ }^{3}$, Rachel L. Johnson, $\mathrm{MS}^{4}$, Rocco Pallin, MPH${ }^{5}$, \\ Matthew Miller, MD, SCD , and Garen J. Wintemute, MD, $\mathrm{MPH}^{5}$
}

'Department of Emergency Medicine, , University of Colorado School of Medicine, Aurora, CO, USA; ${ }^{2}$ Department of Emergency Medicine, , Rhode Island Hospital/Alpert Medical School, Brown University, Providence, RI, USA; ${ }^{3}$ Division of Cardiology, , University of Colorado School of Medicine, Aurora, CO, USA; ${ }^{4}$ Department of Biostatistics \& Informatics, , Colorado School of Public Health, Aurora, CO, USA; 5 Violence Prevention Research Program, Department of Emergency Medicine, Davis School of Medicine, , University of California, Sacramento, CA, USA; ${ }^{6}$ Department of Health Sciences, Bouvé College of Health Sciences, , Northeastern University, Boston, MA, USA.

J Gen Intern Med 34(10):1981-3 DOI: $10.1007 / \mathrm{s} 11606-019-05089-1$

() Society of General Internal Medicine 2019

\section{INTRODUCTION}

The cognitive impairment and behavioral disturbances accompanying dementia can impair individuals' ability to safely operate a car or be home alone; these issues may also increase risk of firearm injury or death, for both persons with dementia (PWD) and caregivers. ${ }^{1}$ Healthcare providers have a recognized role in providing guidance related to both driving and home safety, and materials for providers ${ }^{2}$ and the public ${ }^{3,4}$ exist. Although firearm access in dementia has received increasing attention recently, 5,6 it is unknown whether caregivers of PWD need or want help on this topic, nor is it known how resources on firearm safety compare with driving and home safety for PWD and their caregivers.

\section{METHODS}

We conducted an anonymous internet survey with a convenience sample of family members, friends, or other caregivers (hereafter referred to as "caregivers") of PWD (2/2018-10/ 2018). Eligible participants were English-speaking adults who self-identified as having been a family member, friend, or other caregiver for an individual with dementia within the last 5 years. They were recruited through advertisements on social media, the Alzheimer's Association Trial Match, and a dementia clinic. Because this was a voluntary opt-in survey with advertisements for recruitment, data on non-participants are not available. Participants entered responses directly into REDCap. The Colorado Multiple Institutional Review Board approved this study. Analysis included basic descriptive statistics and review of written comments.

Prior Presentations None.

Published online June 13, 2019

\section{RESULTS}

Eighty-two caregivers in 26 states completed the survey (67\% female, $78 \%$ white, mean age 54 years). The PWD they cared for ( $48 \%$ female, $79 \%$ white, mean age 78 years) mostly lived at home, either alone $(17 \%)$ or with other people (57\%); $36 \%$ of caregivers lived with the PWD. Caregivers reported that $20 \%$ of the PWD drove, $45 \%$ stayed home alone at times, and $24 \%$ had a firearm in the home (45\% with PWD as gun owner). The majority of caregivers identified driving (81\%) and home safety (79\%) as prior, current, or future issues they needed to address with the PWD; 38\% identified firearms as such an issue (Fig. 1). Among the 20 caregivers who had already addressed access to firearms in the PWD's home, $50 \%$ had left the guns in the home but had locked $(n=7)$, unloaded $(n=5)$, and/or disabled $(n=2)$ them; $40 \%$ had removed them from the home to give them to a trusted person $(n=7)$ or law enforcement $(n=2)$ or to sell them $(n=1)$. Only $9 \%$ had spoken with a healthcare provider for advice on firearm safety, compared with $32 \%$ who had done so regarding driving and $38 \%$ regarding home safety (38\%). When asked about "preferred sources of information," most desired information on the internet (written information and videos), healthcare providers, and national and local organizations related to aging and dementia. Only $12 \%$ said they had the necessary information about firearms; one caregiver commented: "The internet was very helpful in all areas, except firearms safety. In fact there was nothing available concerning firearms safety anywhere. It's just not discussed at all."

\section{DISCUSSION}

Although exploratory and small in scope, this survey provides important new information about the experiences and needs of caregivers of PWD as related to safety topics. As expected, driving and home safety concerns were nearly universal, but concern about firearms was also common: half of caregivers identified firearm safety as an issue to address and a quarter of 


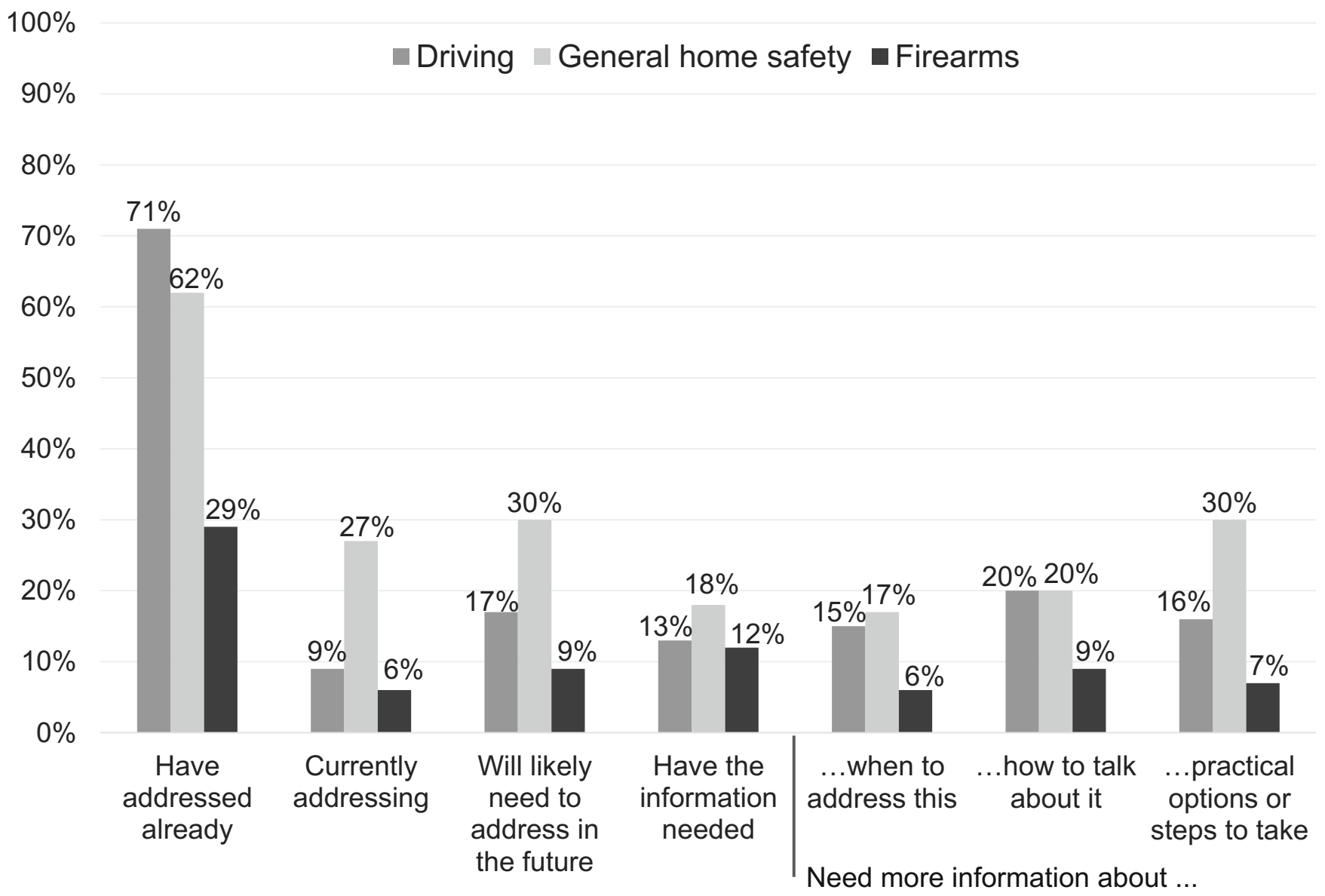

Figure 1 Caregiver views on safety topics to address in context of dementia $(n=82)$. More than one response allowed per participant $($ e.g., "currently addressing" and "will likely need to address in the future").

PWD were reported to live in a home with firearms. Healthcare providers were identified as a preferred source of information more commonly for driving or home safety than firearms, perhaps because conversations about firearms are less commonly initiated by physicians (despite support from national organizations for them to do so), and/or less commonly initiated by PWD and their caretakers. Larger scale studies to better explore firearm storage and discussions in this population will be important to understand the general patterns of firearm access among PWD, including changes over time and the needs of PWD and their caregivers. Future work to better understand the impediments to engaging in conversations about firearm safety with PWD and their caregivers is an important first step towards developing, refining, and disseminating acceptable and effective approaches to enhancing the safety of PWD and those around them while still acknowledging their rights and identities.

Acknowledgments: Ashley Clement assisted with study preparation and posting on Alzheimer's Trial Match. Peter Pressman assisted with participant recruitment.

Corresponding Author: Marian E. Betz, MD, MPH; Department of Emergency Medicine, University of Colorado School of Medicine, $12401 \mathrm{E}$ 17th Ave B215, Aurora, CO 80045, USA (e-mail: marian. betz@ucdenver.edu).
Funders This work was supported by the Paul Beeson Career Development Award Program (The National Institute on Aging; AFAR; The John A. Hartford Foundation; and The Atlantic Philanthropies; K23AG043123); The Heising-Simons Foundation (2016-219; 20170447); the National Institute of Mental Health (RO1HD093655; R34 MH113539); the American Heart Association (18CDA34110026); and the California Wellness Foundation (2014-255).

\section{Compliance with Ethical Standards:}

The Colorado Multiple Institutional Review Board approved this study.

Conflict of Interest: The authors declare that they do not have a conflict of interest.

Disclaimer: Its contents are solely the responsibility of the authors and do not necessarily represent the official views of the funding agencies. No sponsor had any direct involvement in study design, methods, subject recruitment, data collection, analysis, or manuscript preparation.

\section{REFERENCES}

1. Betz ME, McCourt AD, Vernick JS, Ranney ML, Maust DT, Wintemute GJ. Firearms and dementia: Clinical considerations. Ann Intern Med. 2018; 169(1):47-49.

2. American Geriatrics Society, Pomidor A. Clinician's guide to assessing and counseling older drivers (3rd edition). National Highway Traffic Safety Administration Washington, DC; 2016. 
3. Family Conversations with Older Drivers. 2010; http://www.thehartford. com/mature-market-excellence/family-conversations-with-older-drivers. Accessed November 12, 2018.

4. Horvath KJ, Harvey R, Moo LR. A Guide for Families: Keeping the Person with Memory Loss Safer at Home. U.S. Department of Veterans Affairs; 2016; https://www.va.gov/geriatrics/docs/HOME_SAFETY_BOOKLET_81-17.pdf. Accessed February 13, 2019.

5. Hsieh JK, Arias JJ, Sarmey N, Rose JA, Tousi B. Firearms among cognitively impaired persons: A cross-sectional study. Ann Intern Med. 2015;163(6):485-487.
6. Spangenberg KB, Wagner MT, Hendrix S, Bachman DL. Firearm presence in households of patients with Alzheimer's disease and related dementias. J Am Geriatr Soc. 1999;47(10):1183-1186.

Publisher's Note Springer Nature remains neutral with regard to jurisdictional claims in published maps and institutional affiliations. 